\section{The ATLAS experiment}

One of two general-purpose experiments on LHC. The detector layout consists of:

- Inner Detector (ID)

- Pixel Detector

- SemiConductor Tracker (SCT)

- Transition Radiation Tracker (TRT)

- Calorimeters

- Muon Spectrometer

- central solenoid $(2 \mathrm{~T})$

- outer toroids (3.9 T)

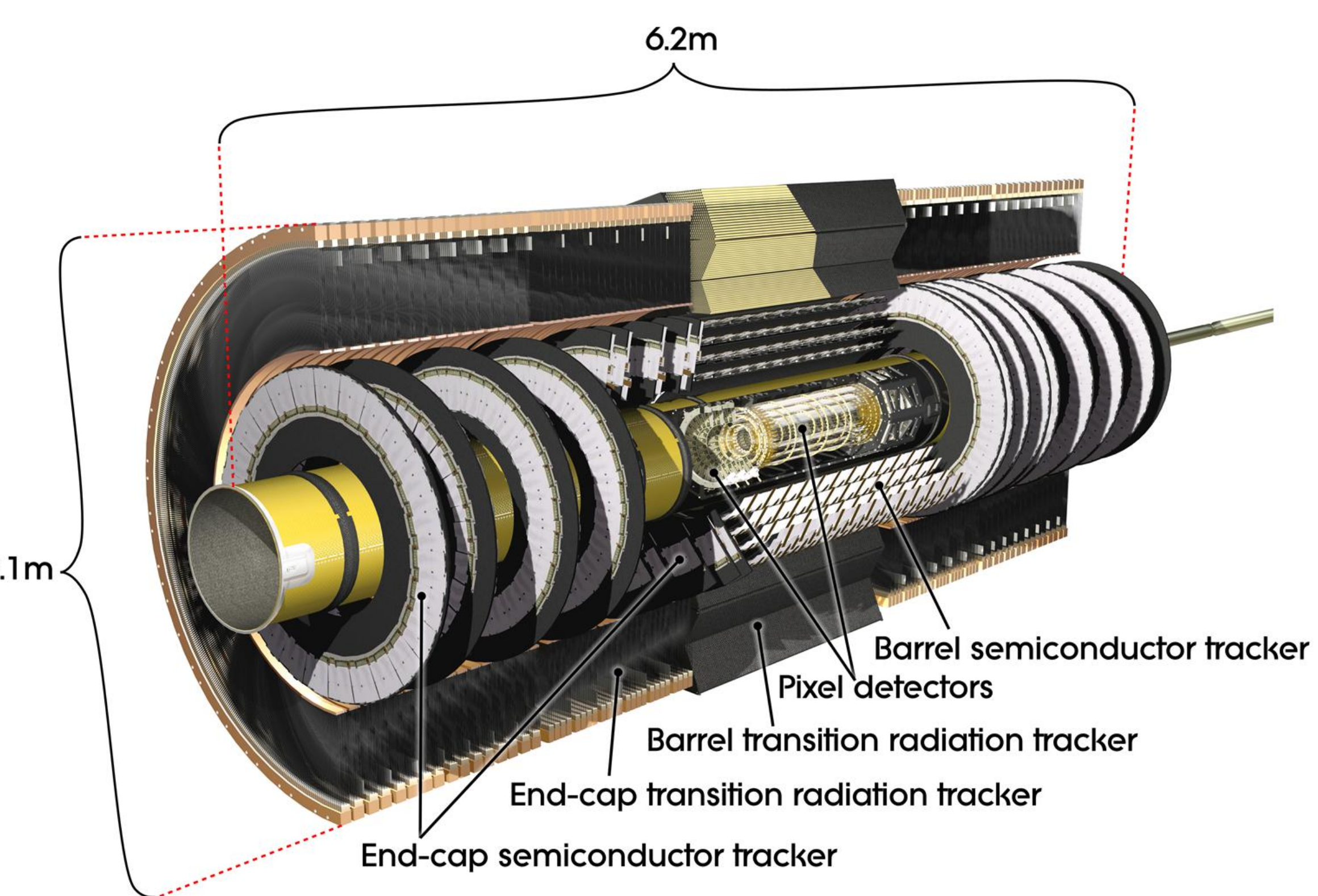

The Semiconductor Tracker (SCT)

\title{
SCT Design
}

4088 modules, 4 barrel cylinders and 18 end-cap disks, 9 on each side

- back-to-back planar sensors with $40 \mathrm{mrad}$ angle

- 6.3 million strips

- up to $500 \mathrm{~V}$ bias voltage (usual $150 \mathrm{~V}$ )

Module specification:

- resolution: $\mathrm{r} \phi \sim 16 \mu \mathrm{m}, \mathrm{z} \sim 580 \mu \mathrm{m}$

- binary readout with optical data transfer

- hit threshold: $1 \mathrm{fC}$

- 12 read-out chips ASIC (ABCD3TA) per module, 128 channels each

- $5.6 \mathrm{~W} /$ module (10 W after 10 years of running)
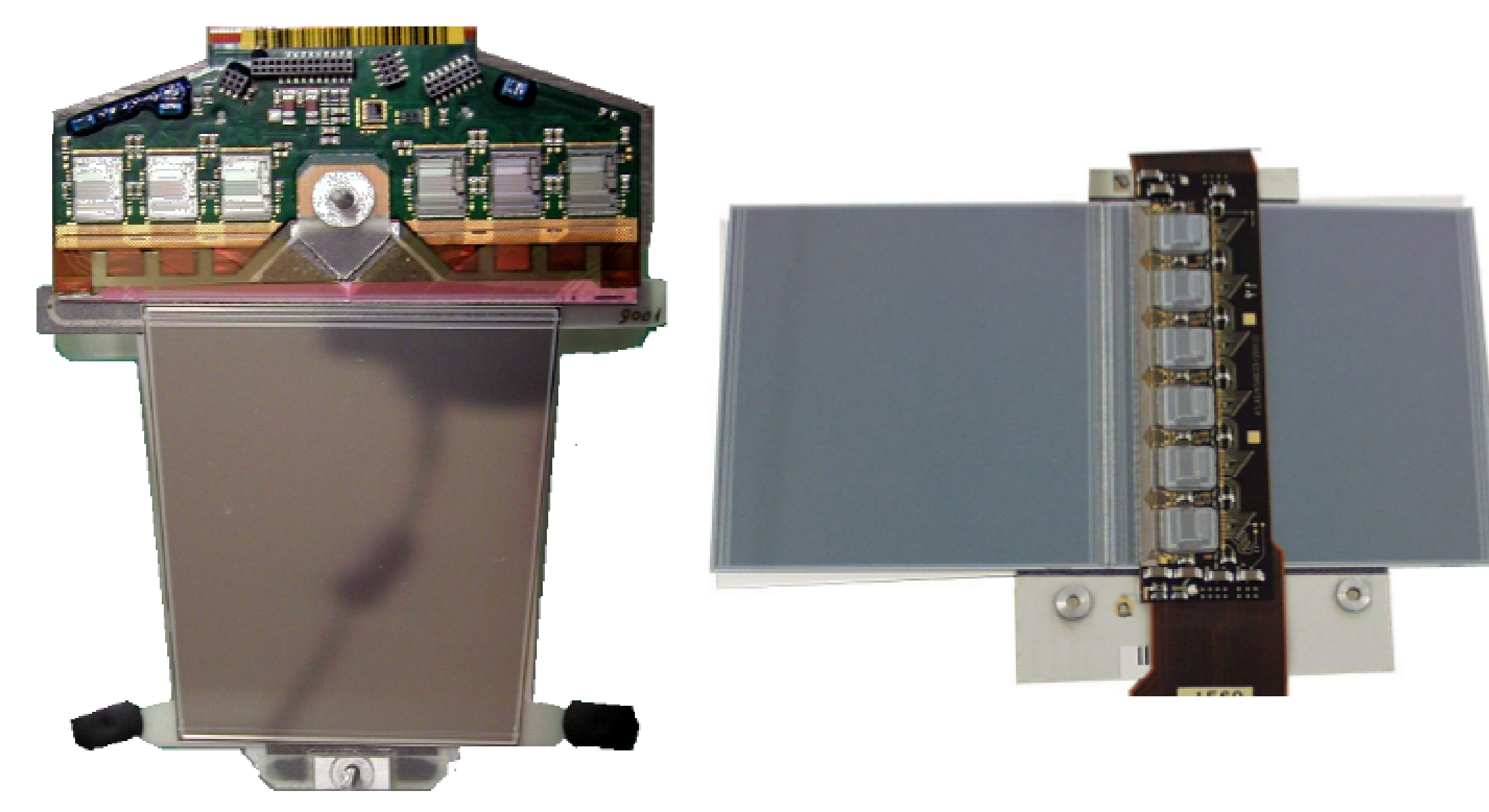

\section{Tracking Performance}

\section{Hit efficiency}

The intrinsic hit efficiency is determined as ratio of the number of recorded hits on track $\left(p_{T}>1 \mathrm{GeV} / \mathrm{c}\right)$ in SCT and the number of possible hits. At least 7 hits are required for SCT standalone tracks and 6 for ID combined tracks. The barrels have a mean hit efficiency of $99.9 \%$ over al layers, for end-caps it is $99.8 \%$

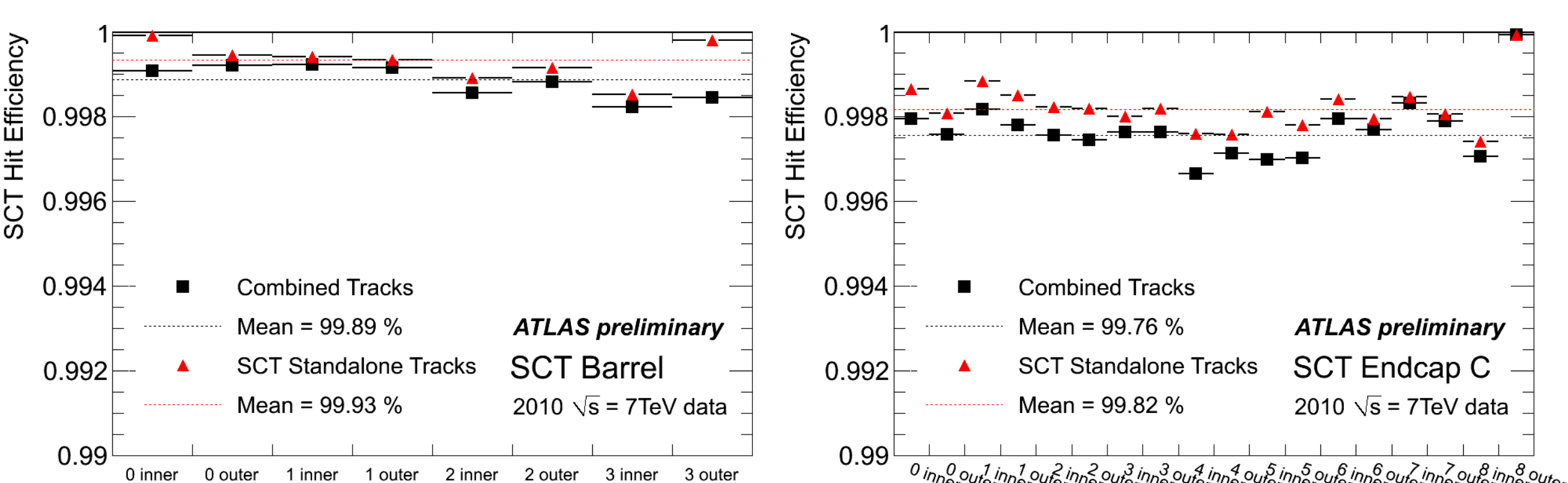

Hit efficiency for barrel and end-cap C, for SCT standalone tracks and combined tracks (use information across all 3 ID systems).

\section{Noise}

Noise is measured in regular calibrations by injecting charge and plotting hits vs. threshold (S-curve) or during collisions as noise occupancy which is defined as fraction of strips with a hit not correlated with activity in the detector (used for monitoring).
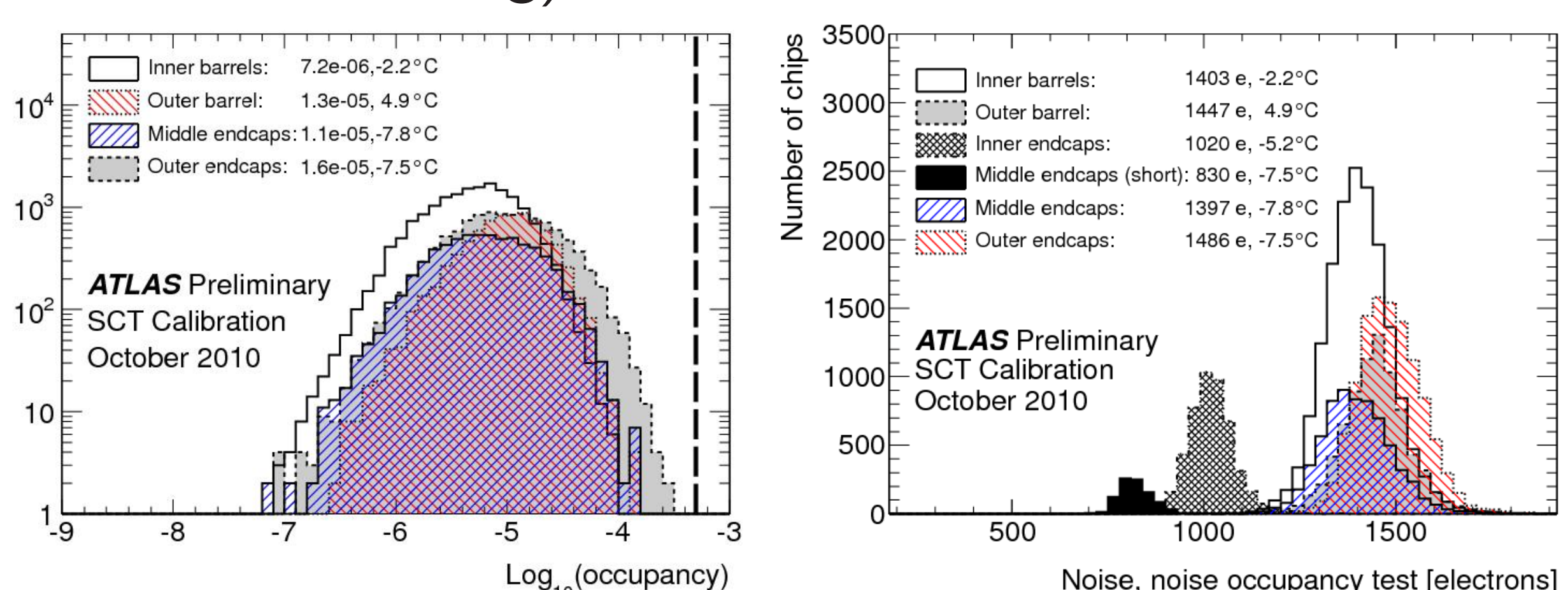

Noise occupancy for the SCT barrels and end-caps (left, dashed line indicates the nominal design limit $\left.5 \times 10^{-4}\right)$ and mean chip noise in $\mathrm{e}^{-}$for different shape of modules (right, to compare with threshold of $\sim 6000 \mathrm{e}^{-}$for tracks).

\section{Lorentz Angle}

The drift direction of charge carriers is deflected in magnetic field by Lorentz force. The Lorentz angle is extracted from minimum of clustersize vs. track angle to module and it depends on the magnetic field, module temperature and depletion voltage. Measured values are in very good agreement with predictions.
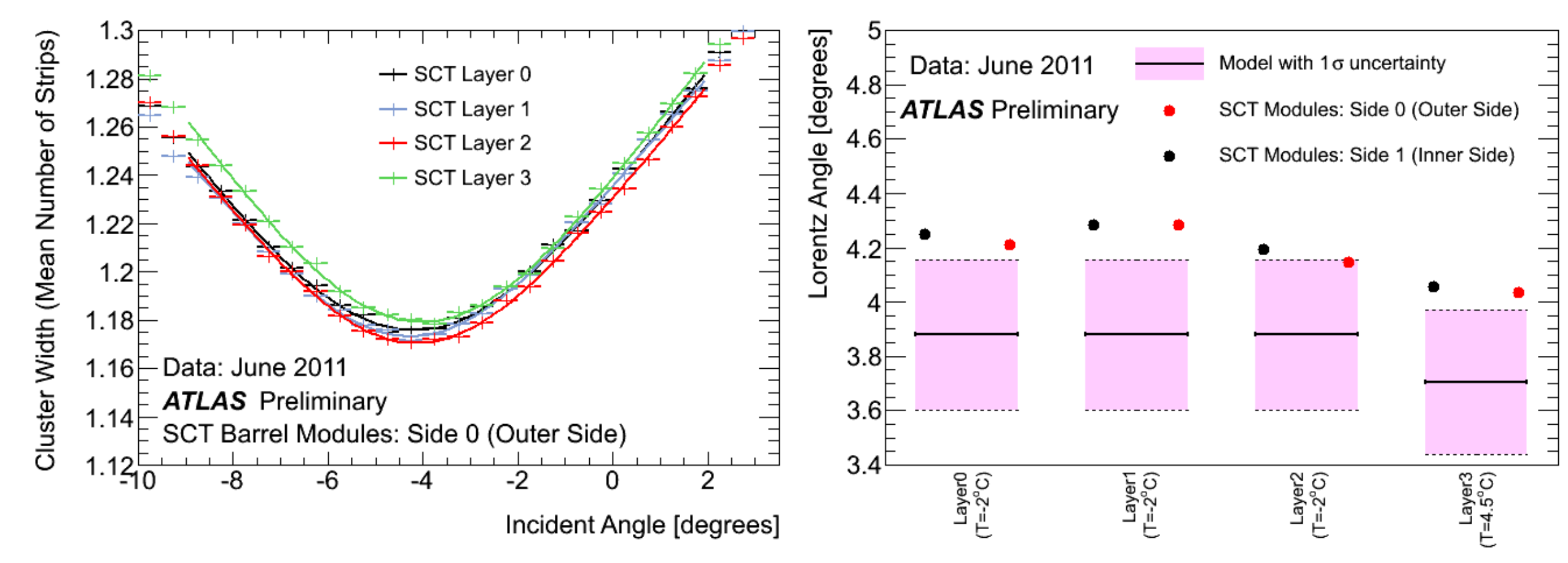

ter width size vs. track incident angle (left) for the barrel layers and

\section{Alignment}

Alignment is performed using a track-based algorithm which minimises the $\chi^{2}$ of track hit residuals (measured hit position minus expected position from track extrapolation). The FWHM of distributions is very close to ideal limits from Monte Carlo predictions.
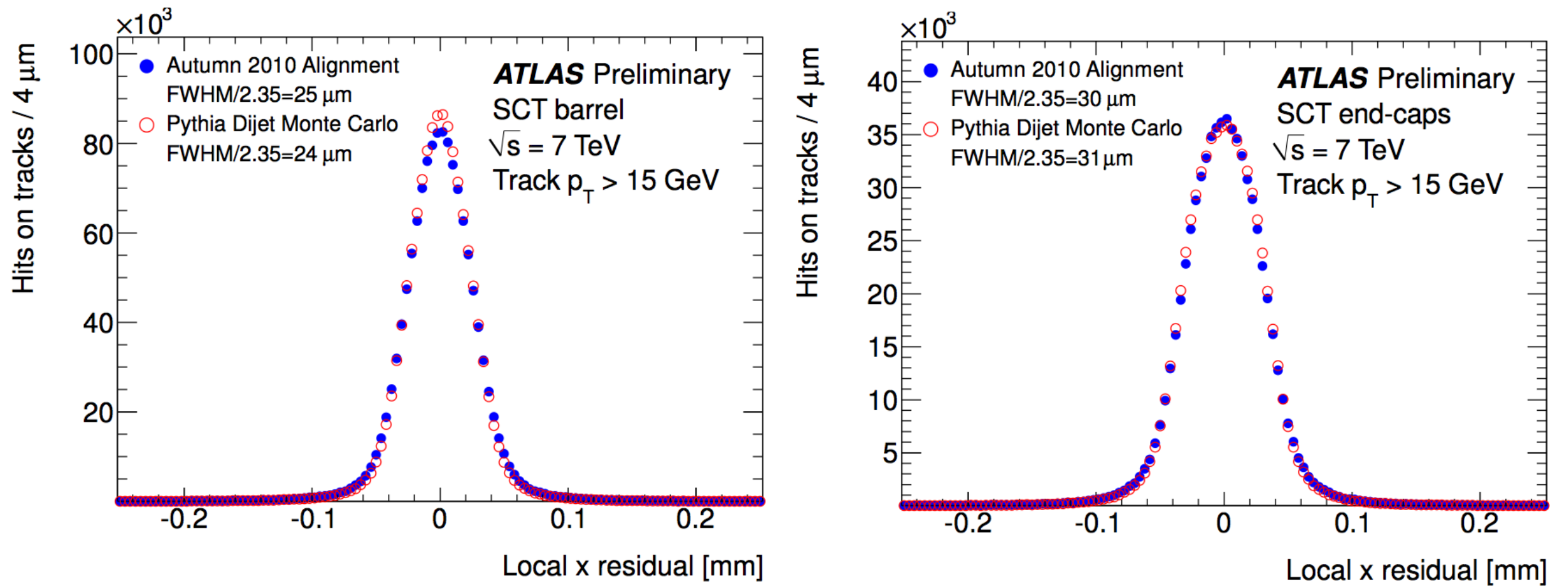

Carlo simulation of
barrel and end-caps.

\section{Radiation damage}

As expected from bulk damage due to non-ionising radiation, we observe a leakage current increase in close correlation with delivered luminosity and sensor temperature. It is in excellent agreement with prediction of Hamburg/Dortmund model (using FLUKA [3] for conversion of collision luminosity to $1 \mathrm{MeV}$ neutron-equivalent fluence and taking into account the self-annealing effects) thus we can expect this excellent performance throughout the expected lifetime of SCT.

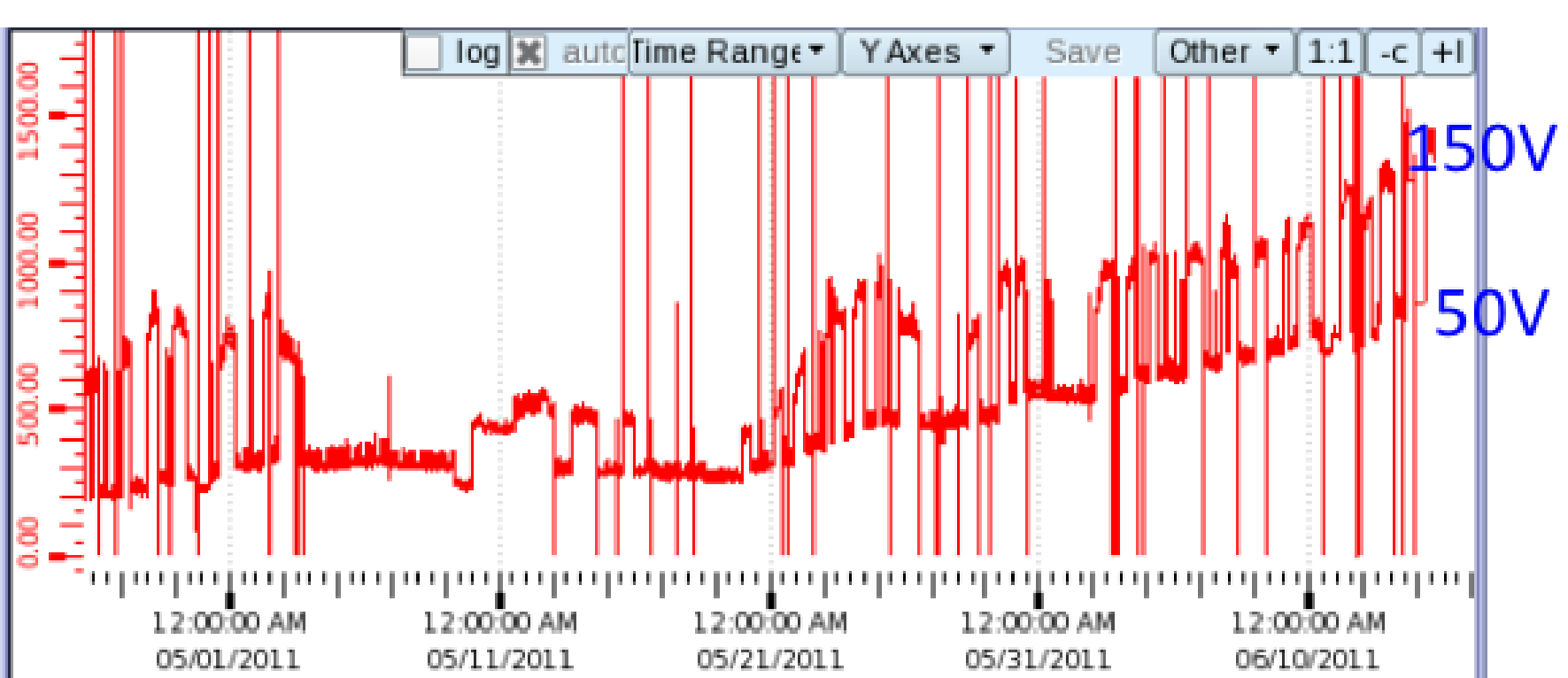

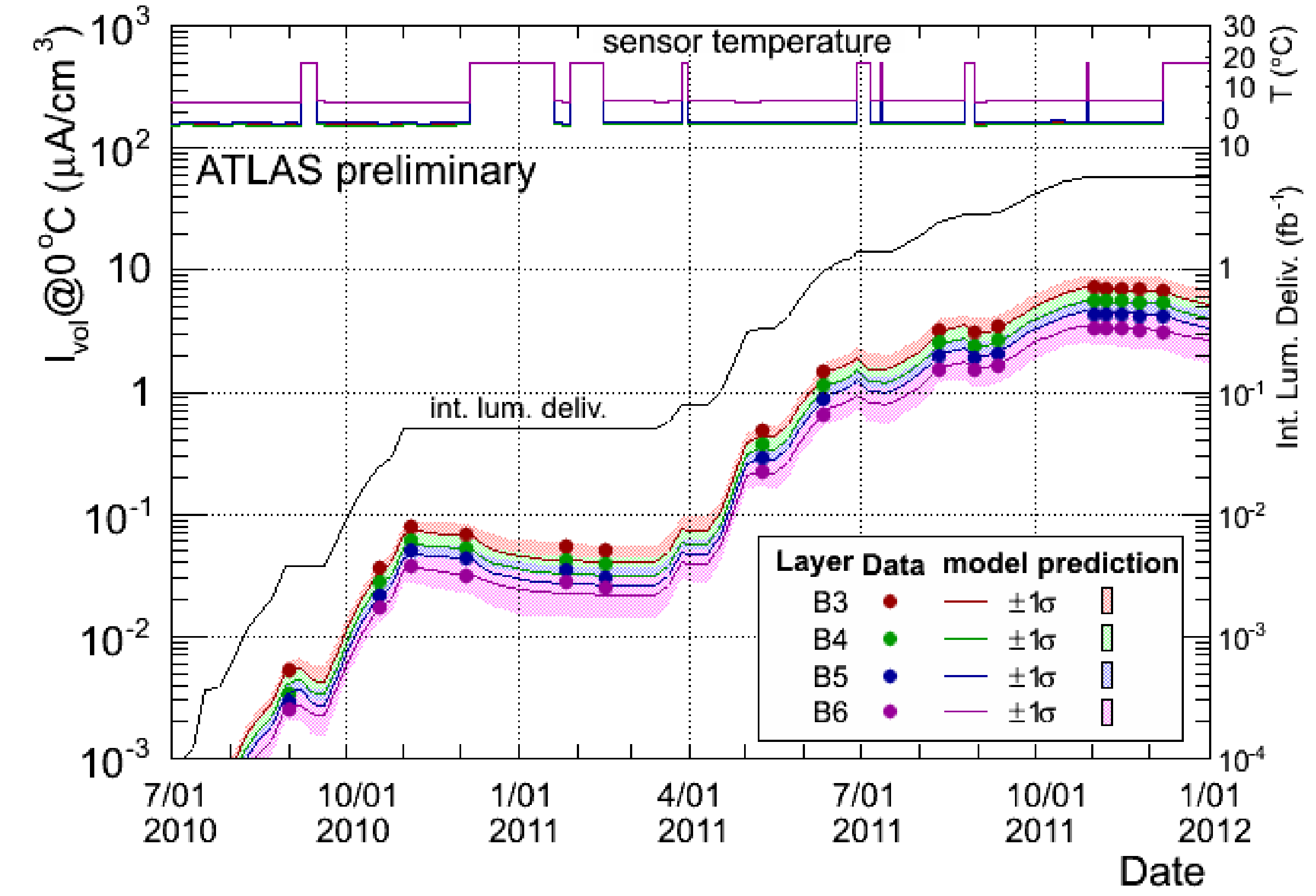

ST barrel leakage currents during 2010 and 2011, showing correlations with delivered luminosity and temperature, and compared to predictions from

\section{Data Taking}

LHC delivered more than $11 \mathrm{fb}^{-1}$ integrated luminosity of p-p collisions in 2010-2012 and two one-month periods of heavy ion collisions. More than $99 \%$ of SCT strips are fully operational. The number of disabled strips or chips (high noise, un-bonded) is negligible and the largest contribution is from typically 30 modules disabled due to various $\mathrm{HV}$, $\mathrm{LV}$, readout and cooling problems.

The SCT data quality is well above design requirement of $99 \%$, it recorded $99.9 \%$ of stable beam collisions in 2010 and $99.6 \%$ in both 2011 and 2012 (until June $18^{\text {th }}$ ).

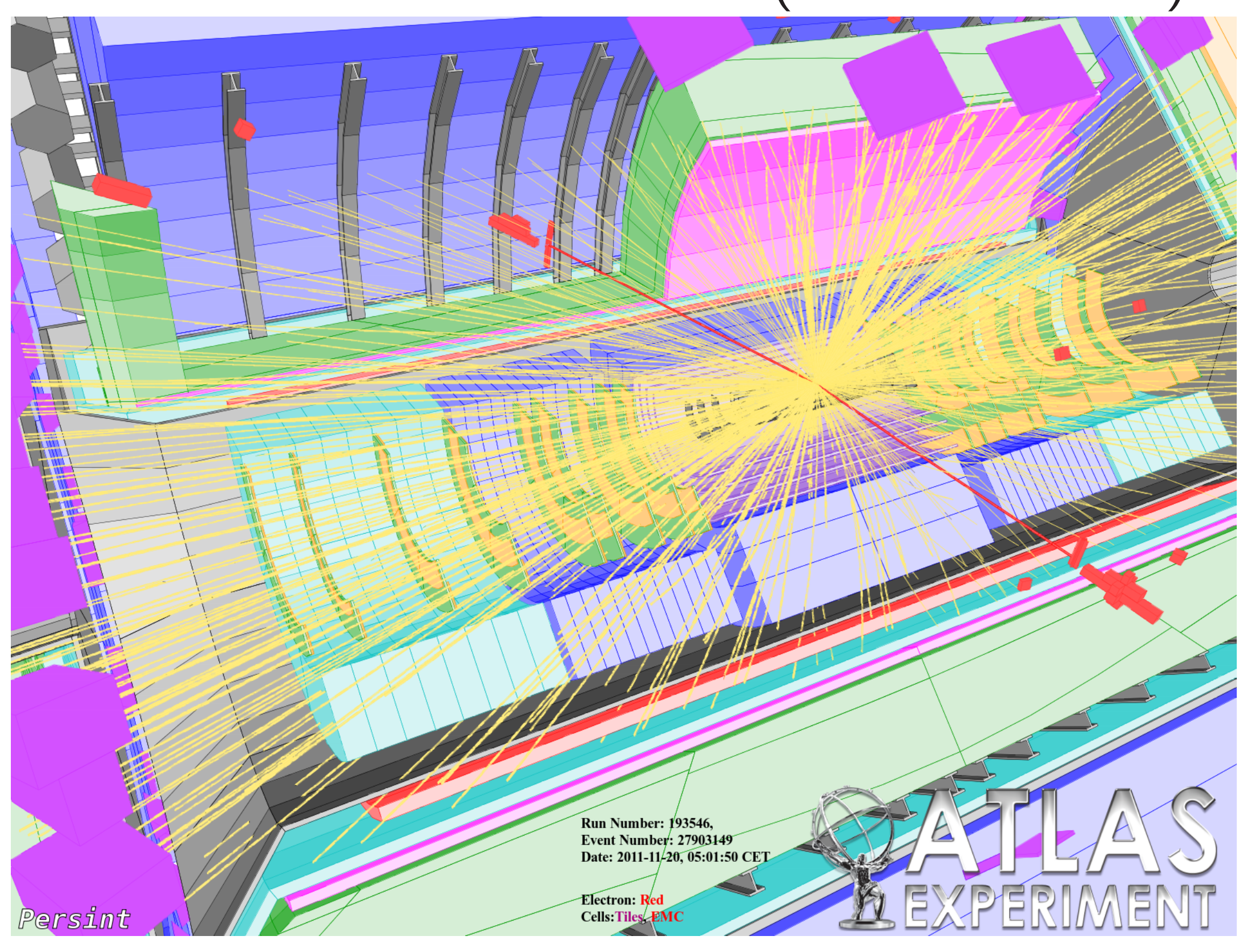

\section{Detector Operation}

\section{Data Acquisition (DAQ)}

- online monitoring of chip errors in the data and automatic reconfiguration of modules leading to low fraction of data links with errors (typically under $0.2 \%$ )

- global reconfiguration every $\sim 30$ minutes (precaution against Single Event Upset - spontaneous corruption of module configuration)

- "stopless" removal and recovery of busy RODs (ReadOut Driver Board)

- possibility to reconfigure whole SCT without the need to stop ATLAS data-taking

- failing off-detector optical transmitters (Tx-plugins) significant improvement achieved through a replacement program and a reduction of the humidity in the environment

\section{Detector Control System (DCS)}

- implemented automatic turn-on (HV from $50 \mathrm{~V}$ to 150 $\mathrm{V})$ after Stable beams are declared based on occupancy, background and collimator position checks

- typical time $\sim 1$ minute to ready for data-taking

- some faulty TEH (Thermal Enclosure Heater-pads between SCT and TRT) require to run outermost barrel at elevated temperature

Plans for technical stop 2013-14

- detector consolidation

- upgrade DAQ for higher luminosity

- installation of a new $\mathrm{C}_{3} \mathrm{~F}_{8}$ ID evaporative cooling plant based on a passive siphon concept

- keep sensors cooled most of the time and monitor detector parameters

\section{References}

[1] The ATLAS Collaboration, The ATLAS Experiment at the CERN Large Hadron Collider, JINST 3508003 (2008).

[2] A. Barr et al, The data acquisition and calibration system for the ATLAS Semiconductor Tracker, JINST 3 P01003 (2008)

[3] A. Ferrari, P. Sala, A. Fasso and J. Ranft, FLUKA: A multi-particle transport code, CERN-2005-10 (2005), INFN/TC-05/11, SLA -R-773. 